\title{
Neutrophil extracellular traps mediate articular cartilage damage and enhance cartilage component immunogenicity in rheumatoid arthritis
}

\author{
Carmelo Carmona-Rivera, ${ }^{1}$ Philip M. Carlucci, ${ }^{1}$ Rishi R. Goel, ${ }^{1}$ Eddie James, ${ }^{2}$ Stephen R. Brooks, ${ }^{3}$ \\ Cliff Rims, ${ }^{2}$ Victoria Hoffmann, ${ }^{4}$ David A. Fox, ${ }^{5}$ Jane H. Buckner, ${ }^{2}$ and Mariana J. Kaplan ${ }^{1}$ \\ 'Systemic Autoimmunity Branch, National Institute of Arthritis and Musculoskeletal and Skin Diseases, NIH, \\ Bethesda, Maryland, USA. ${ }^{2}$ Translational Research Program, Benaroya Research Institute at Virginia Mason, Seattle, \\ Washington, USA. ${ }^{3}$ Office of the Clinical Director, Biodata Mining and Discovery Section, National Institute of Arthritis \\ and Musculoskeletal and Skin Diseases, NIH, Bethesda, Maryland, USA. ${ }^{4}$ Division of Veterinary Resources, Office of the \\ Director, NIH, Bethesda, Maryland, USA. ${ }^{5}$ Division of Rheumatology and Clinical Autoimmunity Center of Excellence, \\ Department of Internal Medicine, University of Michigan, Ann Arbor, Michigan, USA.
}

Rheumatoid arthritis (RA) is characterized by synovial joint inflammation, cartilage damage, and dysregulation of the adaptive immune system. While neutrophil extracellular traps (NETs) have been proposed to play a role in the generation of modified autoantigens and in the activation of synovial fibroblasts, it remains unknown whether NETs are directly involved in cartilage damage. Here, we report a new mechanism by which NET-derived elastase disrupts cartilage matrix and induces release of membrane-bound peptidylarginine deiminase- 2 by fibroblast-like synoviocytes (FLSs). Cartilage fragments are subsequently citrullinated, internalized by FLSs, and then presented to antigen-specific $\mathrm{CD4}^{+} \mathrm{T}$ cells. Furthermore, immune complexes containing citrullinated cartilage components can activate macrophages to release proinflammatory cytokines. HLA-DRB1*04:01 transgenic mice immunized with NETs develop autoantibodies against citrullinated cartilage proteins and display enhanced cartilage damage. Inhibition of NET-derived elastase rescues NETmediated cartilage damage. These results show that NETs and neutrophil elastase externalized in these structures play fundamental pathogenic roles in promoting cartilage damage and synovial inflammation. Strategies targeting neutrophil elastase and NETs could have a therapeutic role in RA and in other inflammatory diseases associated with inflammatory joint damage.

Authorship note: CCR and PMC are co-first authors.

Conflict of interest: The authors have declared that no conflict of interest exists.

Copyright: ( 2020 , American Society for Clinical Investigation.

Submitted: April 20, 2020

Accepted: May 20, 2020

Published: July 9, 2020.

Reference information: /CI Insight. 2020;5(13):e139388.

https://doi.org/10.1172/jici.

insight.139388

\section{Introduction}

Rheumatoid arthritis (RA) is the second most common autoimmune condition, affecting around $1 \%$ of the population worldwide. RA is characterized by inflammation of the synovial joints, bone erosion, and cartilage damage and is associated with high morbidity and mortality. A hallmark of RA is the production of autoantibodies against citrullinated protein antigens, which can be detected before the onset of clinical disease (1). Aggrecan, a major extracellular matrix protein in articular cartilage, has been identified as a potent citrullinated autoantigen in RA (2-5). A recent study reported CD4 ${ }^{+} \mathrm{T}$ cells specific for citrullinated aggrecan in patients with RA, which further implicates adaptive immune responses against citrullinated aggrecan in RA pathogenesis (6). However, the mechanism by which these adaptive immune responses to citrullinated aggrecan are initiated remains unknown.

Previous studies have proposed that fibroblast-like synoviocytes (FLSs) and synovial macrophages substantially contribute to the production of proinflammatory cytokines and enzymes that are responsible for cartilage damage. During the initial phases of the disease, when extensive damage to the joints typically occurs, neutrophils are the most abundant cells in the RA joint, suggesting that these cells play an important role in the initiation of joint damage (7). RA neutrophils display an enhanced capacity to form neutrophil extracellular traps (NETs), which are a meshwork of decondensed chromatin decorated with cytoplasmic and granule proteins externalized to the extracellular space. Studies indicate that these structures can modu- 
late pathogenic innate and adaptive immunity $(8,9)$. Furthermore, during NET formation, potent enzymes that can promote tissue injury are released into the extracellular space. However, whether NETs are directly involved in the orchestration of synovial cartilage damage has not been explored. The purpose of this study was to determine the role of NETs, and enzymes released by these structures, in the degradation of cartilage matrix and in the modulation of synovial and systemic adaptive immunity to modified cartilage structures.

\section{Results}

NETs induce FLSs to upregulate proinflammatory cytokines and degrade aggrecan in vitro. RNA-sequencing analysis of RA FLSs treated for 24 hours with spontaneously generated RA NETs indicated significant upregulation of genes coding for inflammatory cytokines and enzymes involved in cartilage damage and extracellular matrix remodeling, such as $I L 33, I L 11$, and MMP3 (Figure 1A and Supplemental Figure 1A; supplemental material available online with this article; https://doi.org/10.1172/jci.insight.139388DS1). This was supported by quantitative PCR in osteoarthritis (OA) and RA FLSs exposed to NETs for 24 to 48 hours (Figure 1, B-D; and Supplemental Figure 1, B-F). Furthermore, increased levels of IL-11 protein were detected by ELISA in FLS supernatants in the presence of NETs (Supplemental Figure 1G).

Aggrecan is the most abundant structural proteoglycan in human articular cartilage. Aggrecanase-1 (ADAMTS-4) and aggrecanase-2 (ADAMTS-5) are the main enzymes implicated in cartilage degradation, and previous reports suggest that these enzymes are produced by FLSs (10-12). However, lysates and supernatants from OA or RA FLSs were unable to degrade recombinant aggrecan in vitro (Supplemental Figure 2, A and B). In contrast, supernatants from FLSs incubated with NETs cleaved recombinant aggrecan in vitro (Supplemental Figure 2A) and displayed elevated activity levels of aggrecanase-1, when compared with untreated FLSs (Supplemental Figure 2C). Aggrecanase-2 was also detected by Western blot analysis, in supernatants of FLSs incubated with NETs, but not FLSs alone (Supplemental Figure 2D), suggesting that NETs may contain enzymes capable of cleaving aggrecan.

Unexpectedly, NETs isolated from healthy control and RA neutrophils contained aggrecanases-1 and -2 (Supplemental Figure 2, D and F). Aggrecanase activity assay demonstrated that aggrecanases were enzymatically active in NETs but not in FLSs (Supplemental Figure 2E). To further corroborate the presence of aggrecanases in the NETs, an ELISA to detect aggrecanase-1- and aggrecanase-2-DNA complexes was performed. RA synovial fluid (SF) contained significantly higher levels of aggrecanase-DNA complexes when compared with OA SF (Figure 1, E and F), suggesting that aggrecanases contained in NETs may mediate cartilage damage.

Notably, NETs degraded recombinant aggrecan in vitro (Figure 2A). We further corroborated this finding in chondrocyte cultures. Chondroitin sulfate fragments were significantly elevated in supernatants from chondrocytes incubated with NETs for 24 hours as compared with controls (Figure 2B). To assess whether cartilage damage was mediated by NET-bound aggrecanases, we repeated the in vitro degradation assay in the presence of chemical inhibitors. A specific inhibitor of aggrecanases did not inhibit NET-mediated aggrecan degradation, nor did inhibition of neutrophil collagenase (MMP8) (Figure 2, C and D). These data suggest that, despite their presence in NETs, aggrecanases are not responsible for NET-mediated degradation of cartilage matrix proteins.

Neutrophil elastase present in NETs mediates cartilage degradation and promotes peptidylarginine deiminase-2 release by FLSs and citrullination of extracellular matrix proteins. Neutrophil elastase is one of the most abundant enzymes contained in NETs and can cleave a broad spectrum of substrates, including histones (13). Moreover, neutrophil elastase-DNA complexes (a surrogate marker of NETs) and elastase activity were significantly elevated in RA SF when compared with OA SF (Figure 3, A and B). To test the possibility that neutrophil elastase mediates NET-associated degradation of aggrecan, NETs were incubated with recombinant aggrecan in the presence of a specific neutrophil elastase inhibitor (sivelestat). Western blot analysis showed that NET-mediated degradation of aggrecan was attenuated in the presence of sivelestat in a concentration-dependent manner (Figure 3C). Similar effects were observed for other major cartilage proteoglycans, including biglycan and clusterin (Supplemental Figure 3, A and B). To further assess if NET-bound elastase is detrimental to cartilage ex vivo, tibiofemoral joint explants were incubated with recombinant neutrophil elastase or with NETs in the presence or absence of sivelestat for 2 weeks. Joints were then stained with safranin-O and cartilage integrity was scored (Figure 3D). Joints exposed to NETs showed significantly higher eburnation and cartilage loss when compared with untreated joints (Figure 3, E and F). Neutrophil elastase inhibition reduced cartilage loss and eburnation (Figure 3, E and F). Moreover, 
A

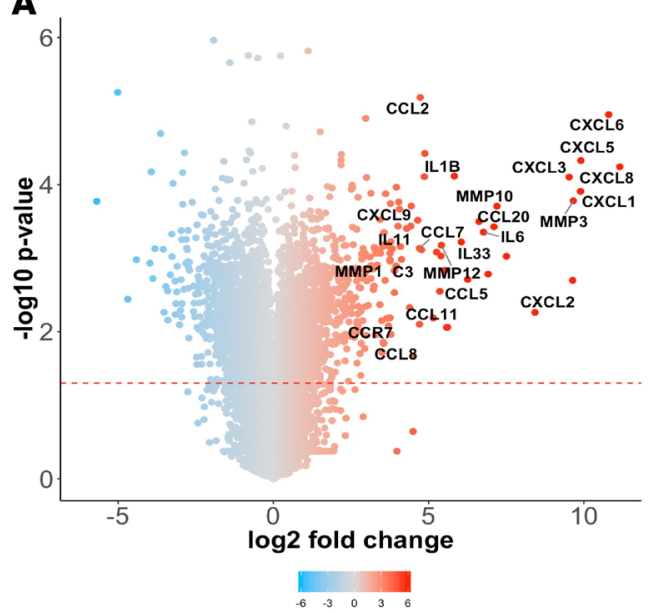

D

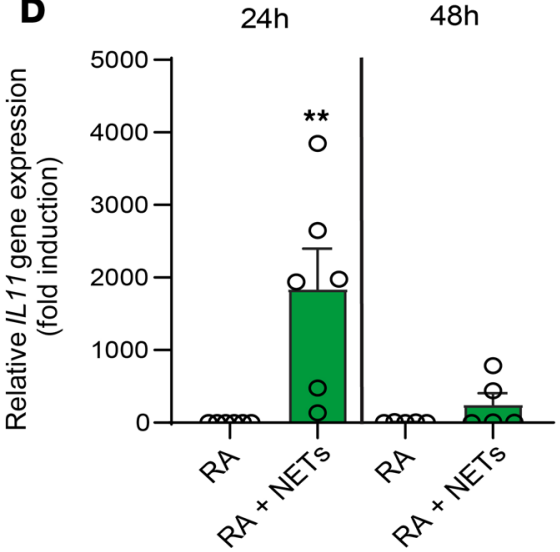

B

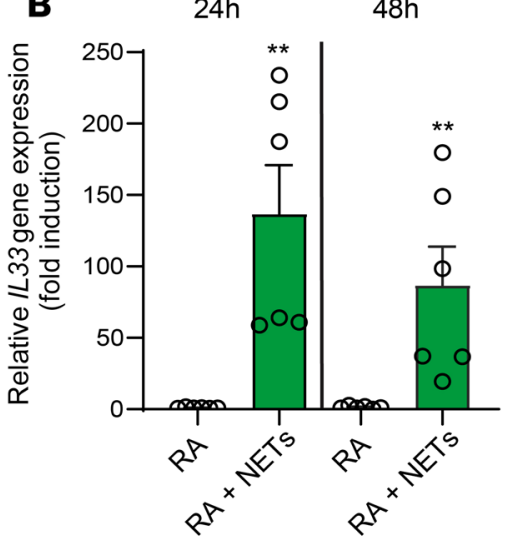

\section{E}

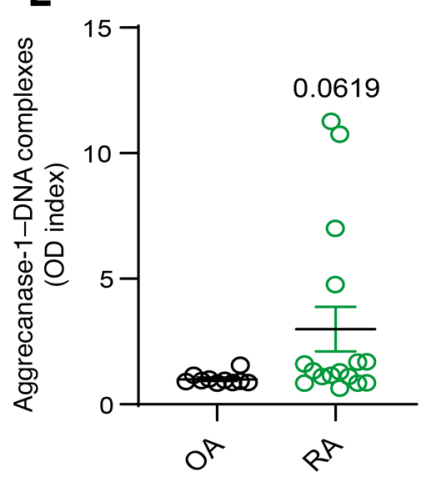

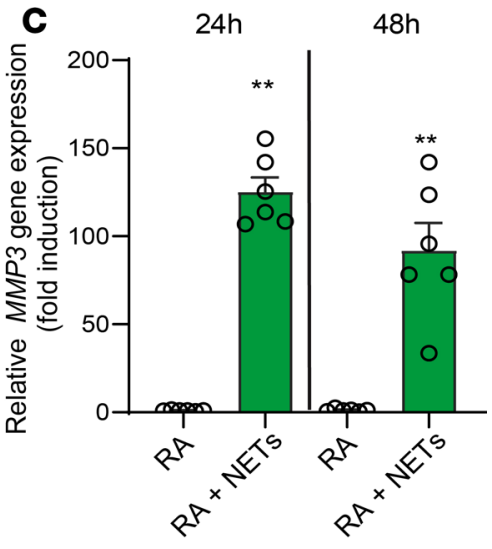

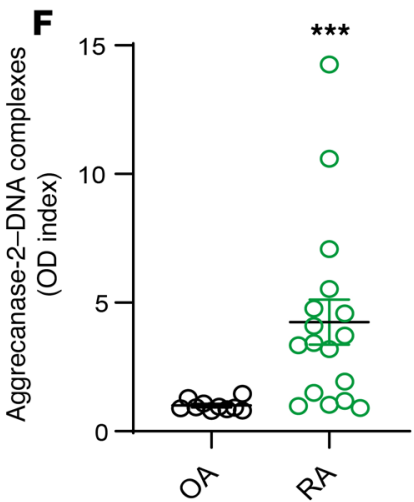

Figure 1. NETs promote a proinflammatory gene signature in FLSs. (A) Volcano plot of differential gene expression comparing NET-treated and untreated RA FLSs. Genes colored in red are upregulated in NET-treated FLSs, while genes colored in blue are downregulated. Genes involved in inflammation and extracellular remodeling are annotated. Quantitative PCR was performed to support differential gene expression of (B) IL33, (C) MMP3, and (D) IL11 and in RA FLSs $(n=6)$ in the presence or absence of added NETs for 24 hours and 48 hours. Results are the mean \pm SEM. Mann-Whitney $U$ test was used. ${ }^{* *} P<$ 0.01. (E) Aggrecanase-1- and (F) aggrecanase-2-dsDNA complexes were measured in synovial fluids (SFs) from osteoarthritis (OA) $(n=10)$ and RA ( $n=17)$ patients. Results are the mean \pm SEM. Mann-Whitney $U$ test was used. ${ }^{* *} P<0.001$.

recombinant neutrophil elastase significantly increased eburnation and cartilage loss in the joints (Figure 3, D-F). Together, these results indicate that neutrophil elastase externalized by NETs has direct effects in cartilage degradation and synovial joint damage.

Peptidylarginine deiminase-2 (PAD2) is involved in citrullination of synovial joint antigens in RA mouse models (14). Quantitative PCR, Western blot, immunofluorescence microscopy, and flow cytometry analyses demonstrated that OA and RA FLSs expressed intracellular PAD2 but not PAD4 (Figure 4, A and B; and Supplemental Figure 3, C-E). PAD2 was also detected in FLS plasma membrane (Figure 4C and Supplemental Figure 3F). Western blot analysis showed that NET-mediated activation of FLSs promotes increased release of PAD2 from the FLS membrane (Figure 4D). This enhanced release of PAD2 was mediated by NET-bound elastase because pretreatment of NETs with an elastase inhibitor decreased PAD2 release from RA FLSs (Figure 4D). In vitro assays showed that aggrecan, biglycan, and clusterin could be citrullinated by PAD2 (Figure 4, E-G). To test whether PAD2 liberated by elastase from FLSs can citrullinate extracellular cartilage proteins, supernatants from FLSs cultured in the presence or absence of NETs were incubated with the same amount of recombinant cartilage proteins. Citrullination assessment demonstrated that aggrecan and biglycan were modified, while clusterin was not, suggesting that only specific glycoproteins can serve as substrates for FLS-derived extracellular PAD2 (Figure 4, E-G). In the presence of NETs, recombinant proteins were citrullinated but also degraded into smaller peptides by neutrophil elastase (Figure 4, 
A

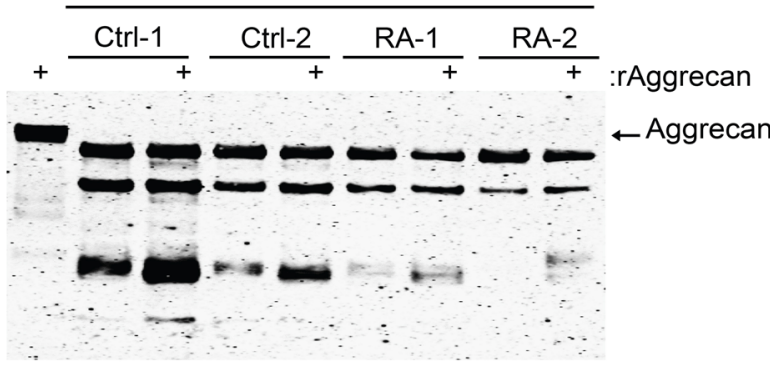

IB: Anti-Aggrecan
B

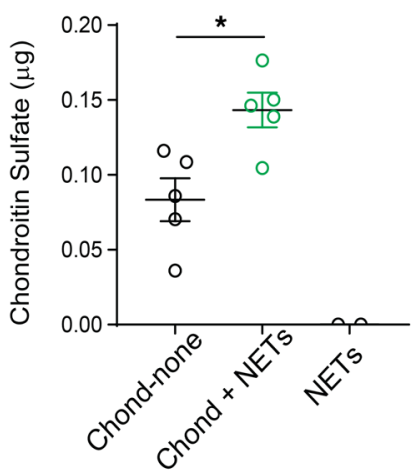

C

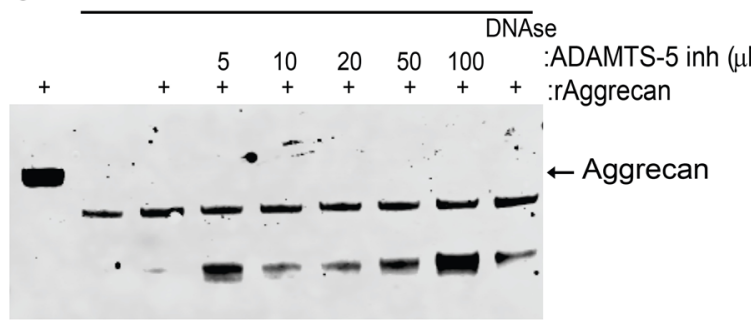

IB: Anti-Aggrecan
D NETs

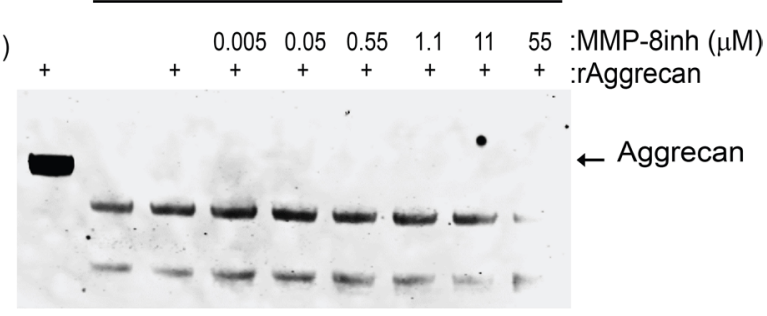

IB: Anti-Aggrecan

Figure 2. NETs degrade aggrecan. (A) Western blot analysis to assess NET-mediated degradation of recombinant aggrecan. (B) Quantification of supernatant release of chondroitin sulfate by chondrocytes incubated in the presence or absence of NETs for 24 hours. Results are the mean \pm SEM of 5 independent experiments. Mann-Whitney $U$ test was used. ${ }^{*} P<0.05$. (C) Western blot analysis to quantify NET-mediated degradation of recombinant aggrecan in the presence of increasing concentrations of ADAMTS-5 inhibitor or DNase I. (D) Western blot analysis to quantify NET-mediated degradation of recombinant aggrecan in the presence of graded concentrations of MMP8 inhibitor.

E-G). These results are consistent with previous studies that have detected PAD2 at elevated levels in the SF of patients with RA and that have shown PAD2 is the main enzyme involved in citrullination in an RA mouse model (14-16).

FLSs present citrullinated cartilage proteins to $C D 4^{+} T$ cells and promote pathogenic adaptive immune responses in humans and mice. We previously showed that FLSs can act as antigen-presenting cells in the synovium, internalize citrullinated NET proteins, and present them to vimentin-specific $\mathrm{CD}^{+} \mathrm{T}$ cells (17). Six citrullinated aggrecan-specific (cit-aggrecan-specific) $\mathrm{CD}^{+} \mathrm{T}$ cell populations were recently described in subjects with RA (6). Based on these findings, we tested the possibility that FLSs can present cit-aggrecan epitopes to $\mathrm{CD} 4^{+} \mathrm{T}$ cell clones. IFN- $\gamma$ production in coculture experiments with FLSs and cit-aggrecan-specific $\mathrm{CD}^{+} \mathrm{T}$ cells demonstrated that FLSs could present cit-aggrecan peptides (Figure 5A). To assess if this enhancement in antigen-specific $\mathrm{T}$ cell responses modulated autoantibody formation, we quantified autoantibodies against cit-aggrecan, cit-biglycan, and cit-clusterin in RA and OA SFs and found them to be enhanced in RA SF (Figure 5, B-D), suggesting that citrullinated versions of cartilage proteins can be immunogenic in $\mathrm{RA}$.

The presence of the HLA-DRB1*04:01 shared epitope associates with risk of developing RA and correlates with $\mathrm{T}$ cell reactivity against citrullinated peptides $(18,19)$. We immunized HLA-DRB*0401 transgenic mice with FLS loaded with NETs and found that they developed significantly increased titers of autoantibodies against cit-aggrecan, cit-biglycan, and cit-clusterin (Figure 5, E-G), suggesting that in the presence of the shared epitope, NETs can promote enhanced adaptive immune responses to citrullinated cartilage antigens in vivo.

Citrullinated cartilage protein-IgG immune complexes activate macrophages to release proinflammatory cytokines. Previous reports indicate that immune complexes (ICs) deposit in RA joints and activate inflammatory responses (20-22). We assessed if ICs containing citrullinated cartilage proteins could directly activate macrophages. Monocyte-derived macrophages incubated with cit-aggrecan or cit-biglycan displayed enhanced release of proinflammatory cytokines, such as TNF- $\alpha$, IL-1 $\beta$, IL-8, and IL-6 (Figure 6, A-D), and this was significantly enhanced in the presence of IgG-ICs. IL- $1 \beta$ and TNF- $\alpha$ are crucial in the orchestration of car- 
A

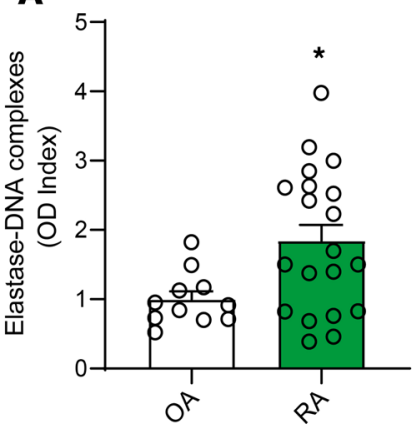

D

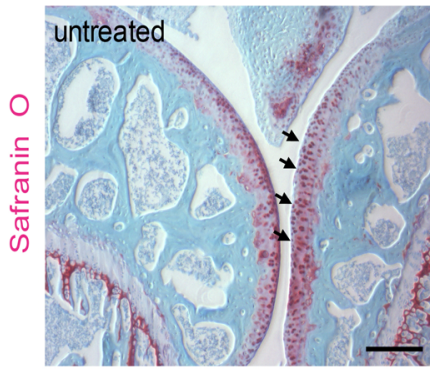

E

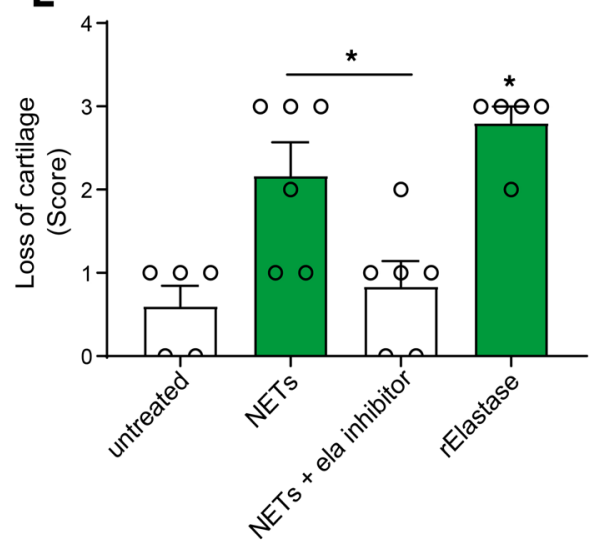

B

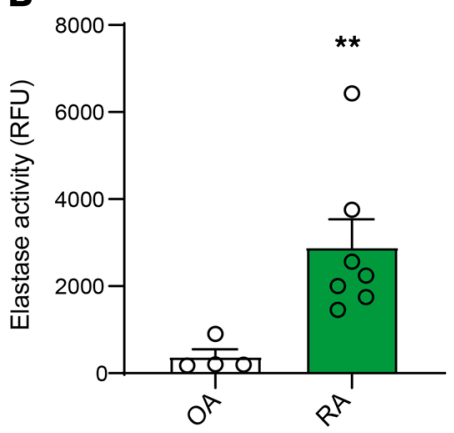

C

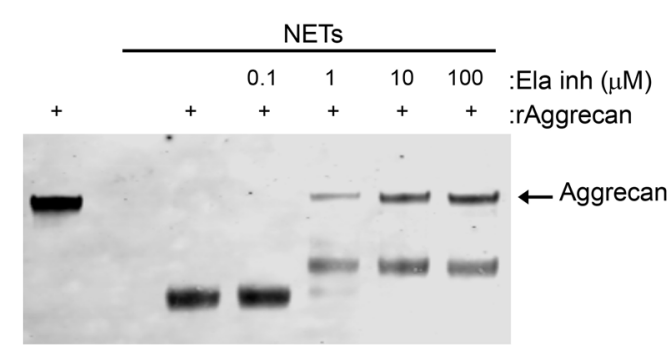

IB: Anti-Aggrecan
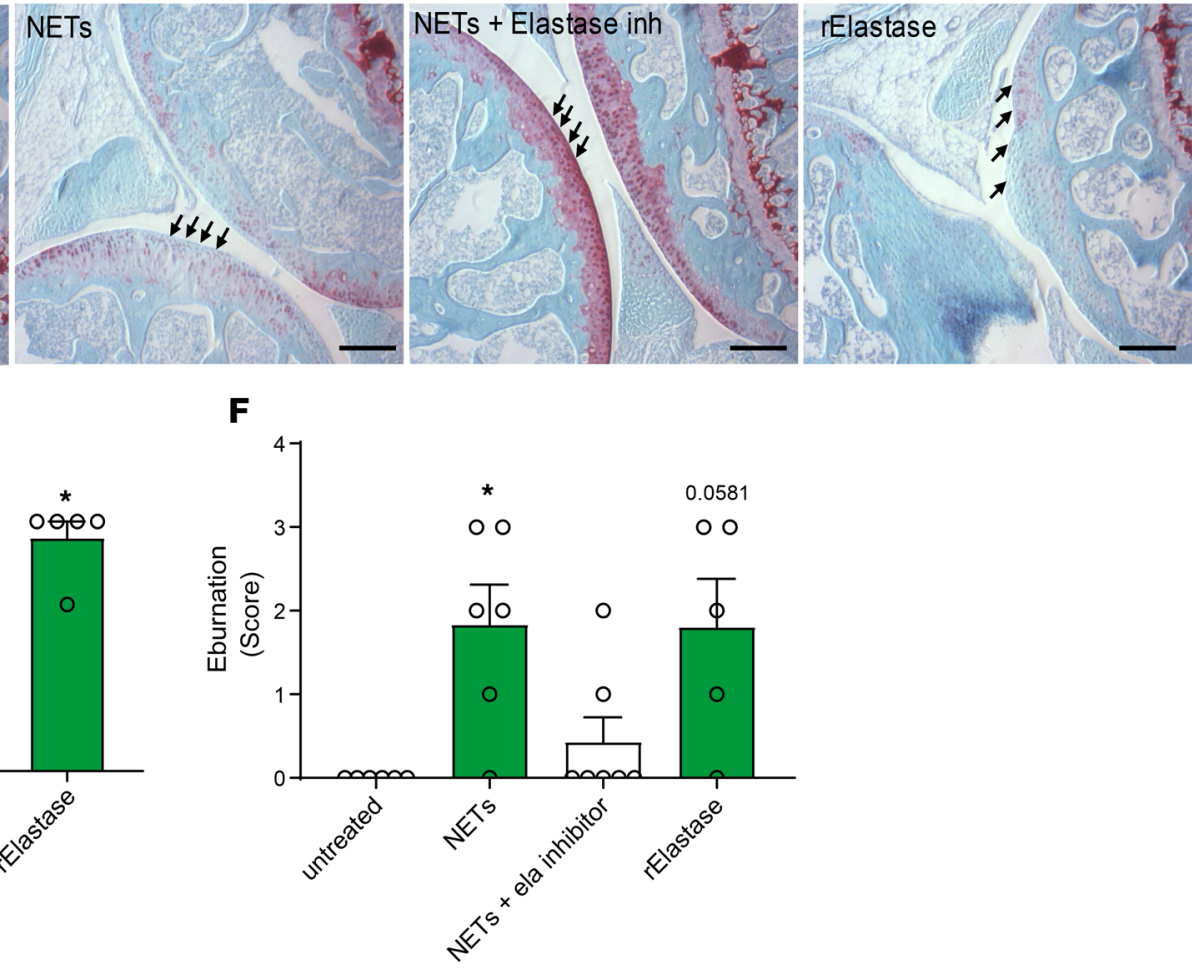

Figure 3. NET-bound neutrophil elastase mediates cartilage degradation. (A) Neutrophil elastase-dsDNA complexes and (B) neutrophil elastase activity were measured in OA $(n=10)$ and RA $(n=17)$ SF. Results are the mean \pm SEM. Mann-Whitney $U$ test was used. (C) Western blot analysis quantifies NET-mediated degradation of recombinant aggrecan in the presence or absence of graded concentrations of neutrophil elastase inhibitor. Results are representative of 3 independent experiments. (D) Safranin- 0 staining and (E) scores of cartilage loss and (F) eburnation in tibiofemoral mouse explants incubated in the presence or absence of NETs, NETs with neutrophil elastase inhibitor, or recombinant neutrophil elastase. Arrows show cartilage areas. Results are the mean \pm SEM of $n=5$ per group. Kruskal-Wallis with post hoc Dunn's test was used. ${ }^{*} P<0.05$, and ${ }^{* *} P<0.01$. Scale bar: $100 \mu \mathrm{m}$.

tilage damage in RA joints and both enhance NET formation $(8,23)$. We hypothesized that these cytokines could amplify cartilage damage by activating neutrophils to release elastase. Supporting this hypothesis, healthy control neutrophils incubated with TNF- $\alpha$ or IL-1 $\beta$ displayed significantly enhanced neutrophil elastase activity in supernatants when compared with untreated neutrophils (Figure 6E).

\section{Discussion}

Increasing evidence supports that neutrophils are important players in the initiation and progression of RA. Recently, NETs have been recognized as key structures in the synovium that orchestrate dysregulated immune responses, inflammation, and joint damage. Interactions between synovial neutrophils and FLSs trigger an inflammatory environment conducive to a pathogenic immune response (17). In this study, we identify a potentially novel pathway of neutrophil-mediated joint destruction, cartilage degradation, and immune activation. Neutrophil elastase present in NETs can directly degrade cartilage components in the 
A

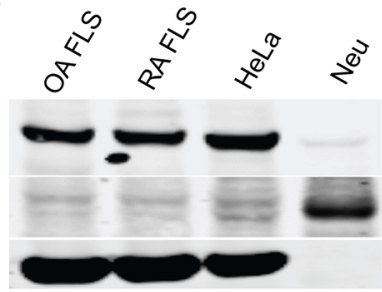

IB: Anti-PAD2

B: Anti-PAD4

IB: Anti-Tubulin

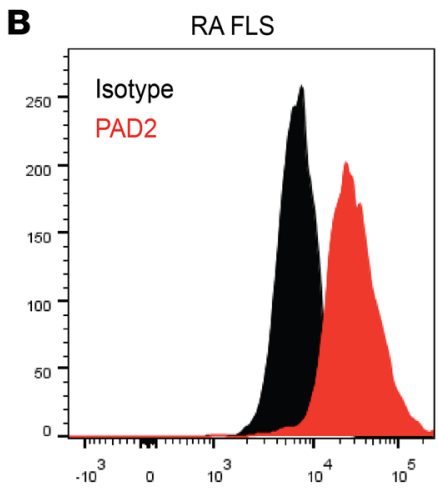

E

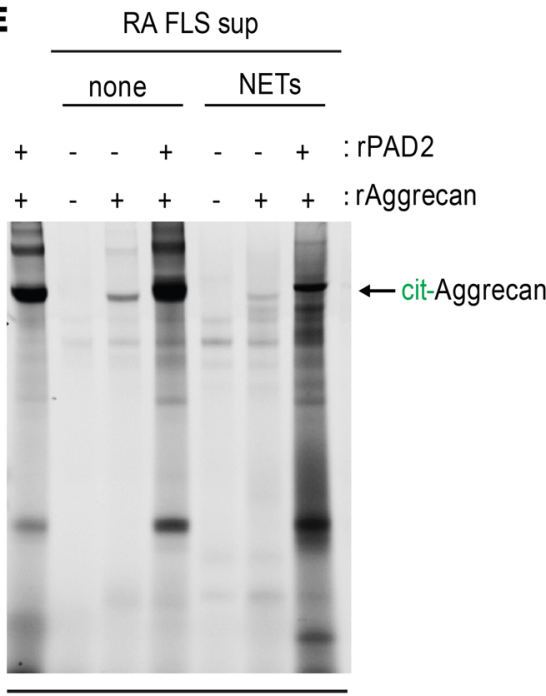

cit-Rh-PG
C
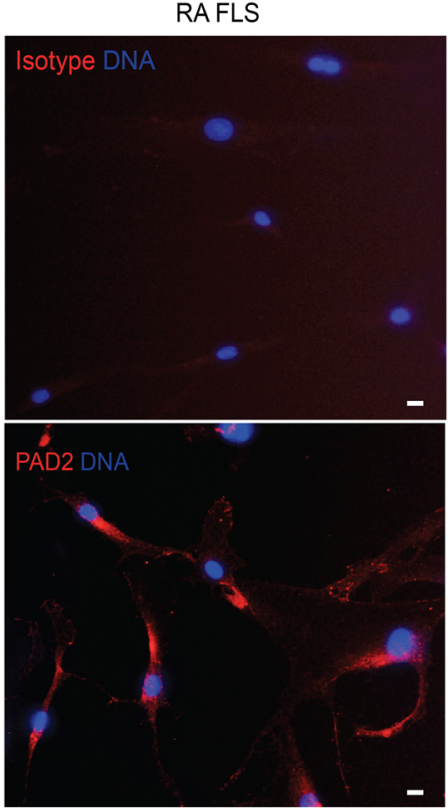

$\mathbf{F}$
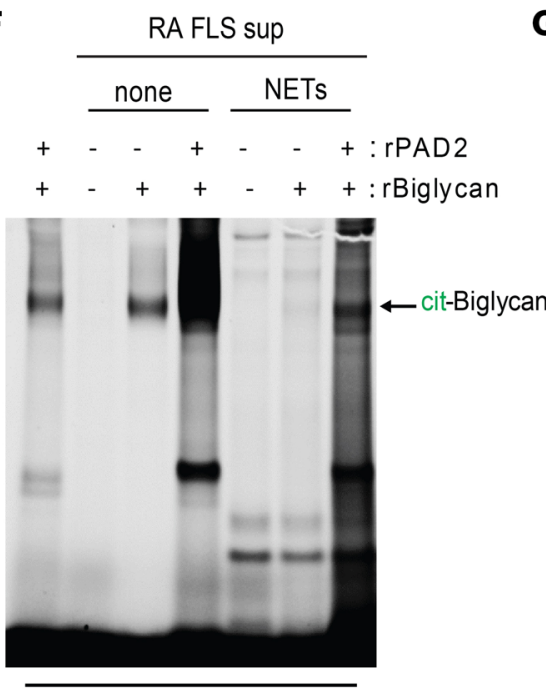

cit-Rh-PG
D

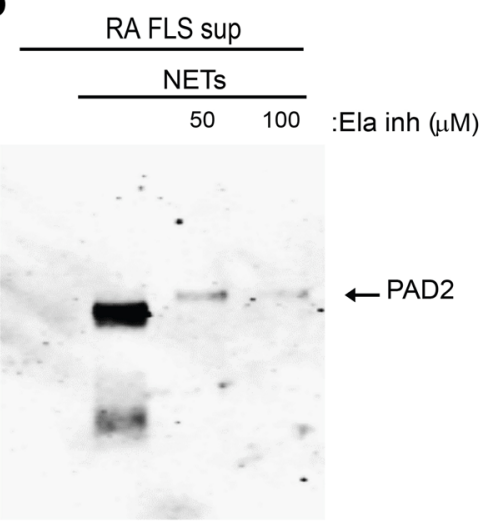

IB: Anti-PAD2
G

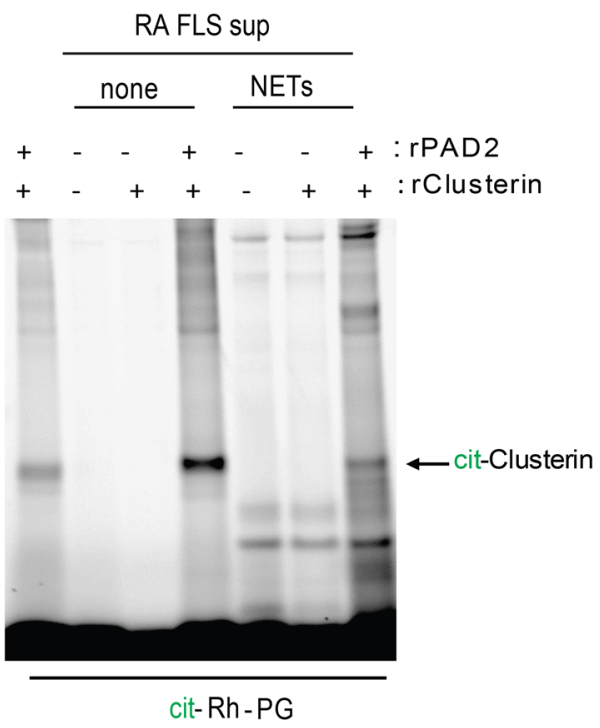

Figure 4. NET-bound neutrophil elastase promotes the release of PAD2 by FLSs and citrullination of extracellular matrix proteins. (A) Western blot analysis quantifying PAD2 and PAD4 expression in OA and RA FLSs. HeLa cells and neutrophil extracts were used as positive controls for PAD2 and PAD4 respectively. Tubulin was used as a loading control. (B) PAD2 expression in RA FLSs by flow cytometry analysis. (C) Representative immunofluorescence of PAD2 expression in the membrane of RA FLSs. (D) Detection of PAD2 in the supernatants of RA FLSs incubated with NETs in the presence or absence of neutrophil elastase inhibitor. PAD2 activity was measured by detecting citrullinated (E) aggrecan, (F) biglycan, and (G) clusterin using Rh-PG citrulline probe in RA supernatants in the presence or absence of NETs. Recombinant PAD2 was used as a control. Results are representative of 3 independent experiments. Scale bar: $10 \mu \mathrm{m}$.

synovium, while amplifying inflammatory pathways in FLSs and macrophages that can further promote joint damage (Figure 7). In addition to their proinflammatory effects, NETs induce PAD2 release by FLSs through a neutrophil elastase effect that enhances citrullination of cartilage proteins (Figure 7). Citrullinated matrix proteins are subsequently internalized by FLSs and can activate antigen-specific $\mathrm{T}$ cells to induce specific autoantibody responses. Furthermore, ICs of citrullinated cartilage matrix antigens can further amplify aberrant, deleterious proinflammatory immune responses (Figure 7).

Aggrecanase-1 and -2 are the main enzymes known to be associated with cartilage damage $(24,25)$. These enzymes have been considered to be released by FLSs in the synovium $(25,26)$. To our knowledge, 
A
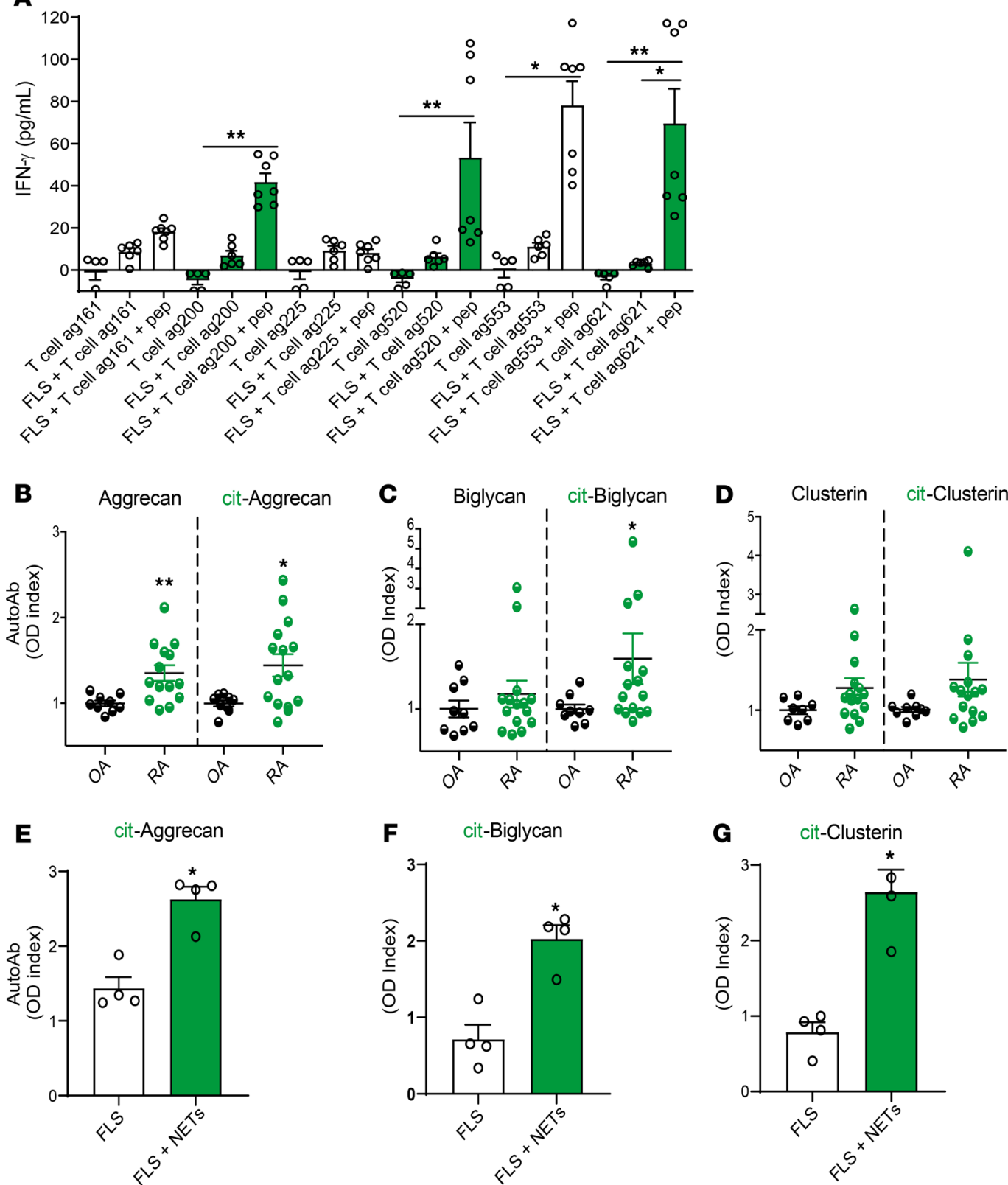

Figure 5. FLSs exposed to NETs present citrullinated cartilage proteins to CD4 ${ }^{+} \mathrm{T}$ cells and promote in vitro and in vivo adaptive immune responses to citrullinated cartilage peptides in humans and mice. (A) IFN- $\gamma$ was measured in supernatants of RA FLSs cocultured for 5 days with cit-aggrecan-specific CD4 ${ }^{+} T$ cells in the presence or absence of cit-aggrecan peptides. Results are the mean \pm SEM of 5 independent experiments. Kruskal-Wallis with post hoc Dunn's test was used. Autoantibodies against native and citrullinated forms of (B) aggrecan, (C) biglycan, and (D) clusterin were measured in OA $(n=9)$ and RA $(n=15)$ SF. Results are the mean \pm SEM. Mann-Whitney $U$ test was used. Sera from HLA-DRB1*04:01 transgenic mice immunized with FLSs or FLSs in the presence of NETs were analyzed for the presence of autoantibodies against citrullinated versions of $(\mathbf{E})$ aggrecan, $(\mathbf{F})$ biglycan, and $(\mathbf{C})$ clusterin. Results are the mean \pm SEM of $n=5$ per group. Mann-Whitney $U$ test was used. ${ }^{*} P<0.05$, and ${ }^{*} P<0.01$.

we describe for the first time that neutrophils express aggrecanase- 1 and -2 , which are released during NET formation. However, these enzymes were not responsible for neutrophil-mediated cleavage of aggrecan. Instead, we found that neutrophil elastase released by NETs is a key player in synovial cartilage degradation. This is consistent with previous reports that activated neutrophils can directly damage cartilage (27, 28) and that RA neutrophils have an activated phenotype that favors NET formation and externalization of neutrophil elastase in the synovium (8). 
A

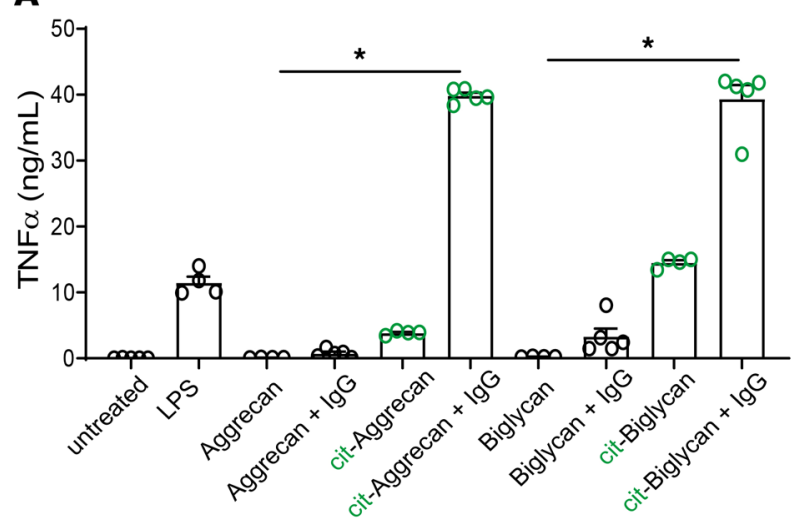

C

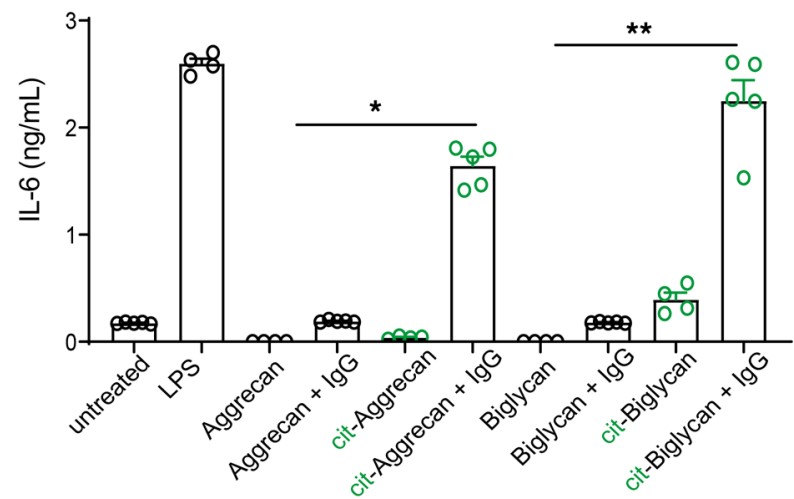

E

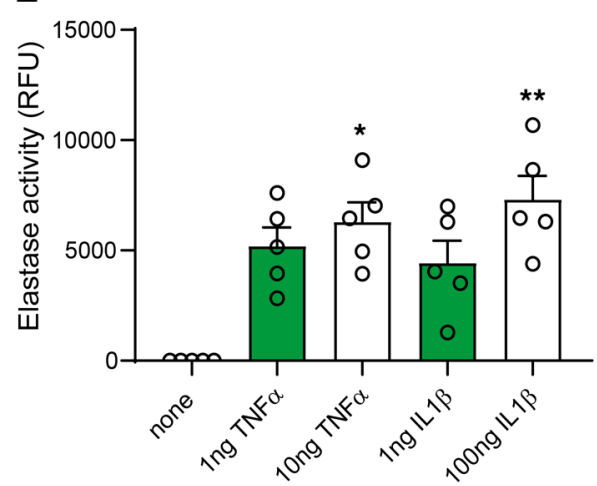

B

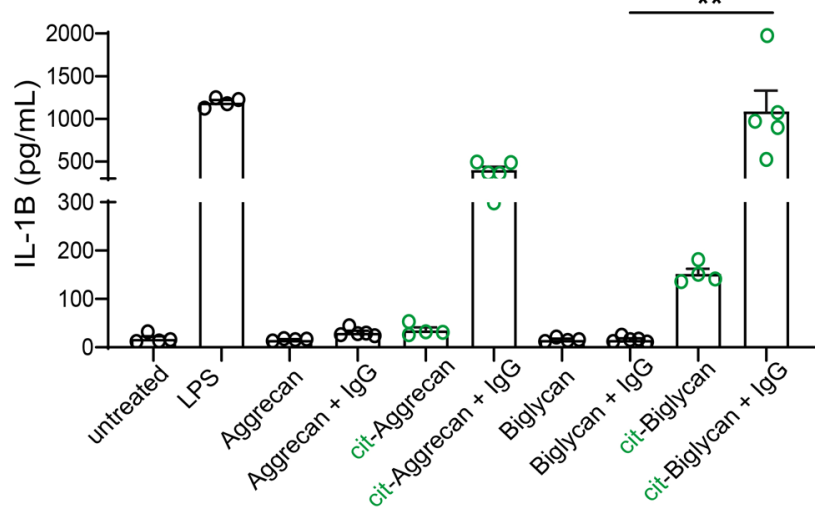

D

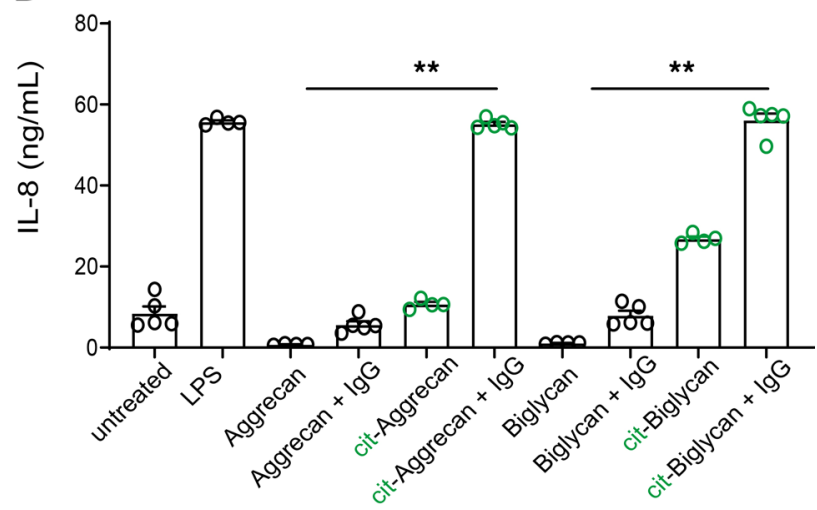

Figure 6. Citrullinated cartilage protein-IgG ICs activate macrophages to release proinflammatory cytokines. M1 macrophages were incubated with native or citrullinated cartilage proteins and ICs for 24-72 hours. Supernatants were analyzed for (A) TNF- $\alpha$, (B) IL-1 $1 \beta$, (C) IL-6, and (D) IL-8. Results are the mean \pm SEM of 4-5 independent experiments. Kruskal-Wallis with post hoc Dunn's test was used. ${ }^{*} P<0.05$, and ${ }^{*} P<0.01$. (E) Supernatants of control neutrophils were analyzed for elastase activity after incubation with TNF- $\alpha$ or IL-1 $\beta$. Results are the mean \pm SEM of 5 independent experiments. Kruskal-Wallis with post hoc Dunn's test was used. ${ }^{*} P<0.05$, and ${ }^{* *} P<0.01$.

Furthermore, patients with RA develop antibodies against citrullinated cartilage fragments, such as aggrecan (6). We now describe a mechanism whereby neutrophils interact with chondrocytes and FLSs, leading to the generation of citrullinated cartilage fragments that can be presented to synovial CD4 ${ }^{+} \mathrm{T}$ cells to promote adaptive immune responses and propagate inflammation. Although this may not be the only mechanism leading to the generation of anti-citrullinated cartilage fragment antibodies, it provides more evidence for the increasingly recognized detrimental role of neutrophils in RA synovium during early and/ or active phases of the disease.

IC deposition is a prominent feature in RA synovium (22). We found that anti-citrullinated cartilage antibodies can form ICs with autoantigens present in the synovium and activate macrophages to release elevated levels of proinflammatory cytokines. TNF- $\alpha$ and IL-1 $\beta$ have been previously implicated in cartilage 


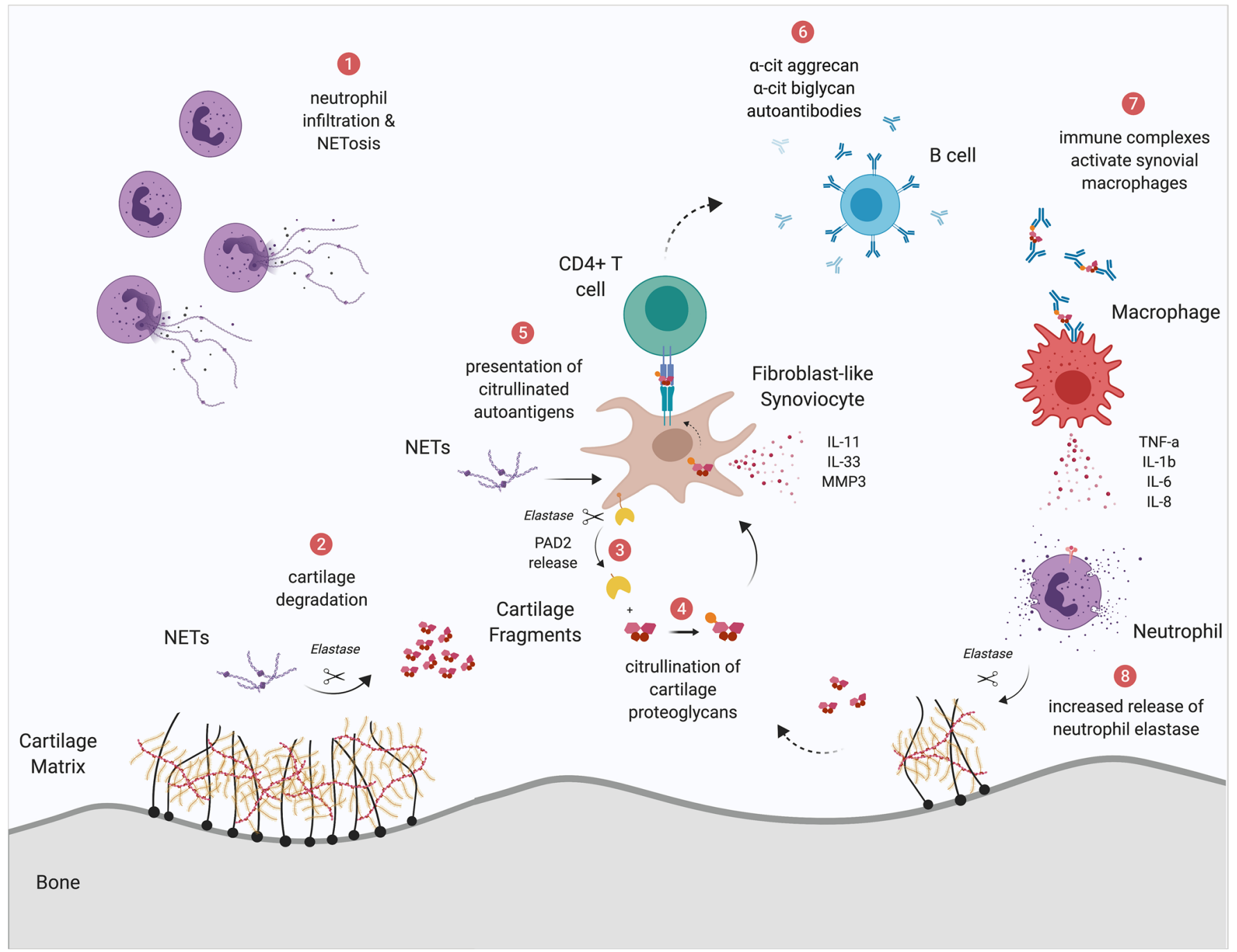

Figure 7. Schematic representation of the role of neutrophil elastase in synovial cartilage integrity in RA. (Number 1) Synovial neutrophils display enhanced NET formation. (Number 2) NETs containing elastase cleave cartilage, generating fragments. (Number 3) Also, NET-derived elastase cleaves FLS-PAD2 off the plasma membrane. (Number 4) Released PAD2 citrullinates cleaved cartilage fragments that are taken up by FLSs. (Number 5) Citrullinated cartilage fragments are presented to specific CD4+ $T$ cells and (Number 6 ) elicit antibody production against citrullinated aggrecan and biglycan by B cells. (Number 7) These autoantibodies form ICs that activate macrophages to release proinflammatory cytokines, such as TNF- $\alpha$, IL-1 $\beta$, IL-6, and IL-8. (Number 8) Proinflammatory cytokines activate neutrophils to release more elastase, creating a vicious cycle that is detrimental to the cartilage.

damage $(29,30)$. We found that these cytokines activate neutrophils to release elastase, promoting cartilage damage and perpetuation of an inflammatory cycle that exacerbates joint destruction.

During NET formation, PAD4 citrullinates RA-relevant autoantigens $(8,31,32)$. Some studies have suggested that PAD2 is a main driver of citrullination, at least in murine models (14). Supporting this finding, we found that FLSs exclusively express PAD2, and it is responsible for citrullinating cartilage fragments generated by neutrophil elastase in the synovium.

Our results indicate that therapies that target NETs or, more specifically, neutrophil elastase could potentially slow the progression of cartilage damage and reduce disability in RA. Our data also highlight the potential role that neutrophils play in damaging other matrix-rich tissues.

\section{Methods}

Antibodies, recombinants, and chemicals. The following reagents were used in this study: recombinant human aggrecan (G1-IGD-G2) (BioLegend), biglycan (Abcam), clusterin (ProSpec), and PAD2 (MilliporeSigma). Aggrecanases' inhibitor (ADAMTS-5) was purchased from MilliporeSigma, MMP8 inhibitor was from Chem Cruz, and neutrophil elastase inhibitor (sivelestat) was purchased from MilliporeSigma. Rabbit anti- 
aggrecanase-1 (Thermo Fisher Scientific, PA1-1750), anti-aggrecanase-2 (Thermo Fisher Scientific, PA11751A), anti-PAD2 (Proteintech, 12110-1-AP), anti-myeloperoxidase (Dako, A0398), mouse anti-tubulin (MilliporeSigma, T5168), anti-PAD4 (LifeSpan Biosciences, LS-C174028-100), anti-clusterin (Santa Cruz Biotechnology, clone B-5, sc-5289), anti-aggrecan (Abcam, clone 6-B-4,ab3778), and anti-Flag (Thermo Fisher Scientific, MA1-91878) antibodies were used in this study.

Human subjects. Samples were obtained from subjects who fulfilled the American College of Rheumatology criteria for RA (33) or were diagnosed with OA based on characteristic clinical and radiographic features and confirmed by pathological findings at joint surgery. Healthy controls were recruited by advertisement. All individuals gave written informed consent and enrolled in a protocol approved by the National Institute of Arthritis and Musculoskeletal and Skin Diseases/National Institute of Diabetes and Digestive and Kidney Diseases Institutional Review Board (IRB; protocol 01-AR-0227) or the University of Michigan Medical IRB (protocol HUM00043667 or HUM00045058). Peripheral blood (PB) was obtained by venipuncture and collected in EDTA-containing tubes. PB was fractionated via Ficoll-Paque Plus (GE Healthcare) gradient. SF was collected during medically necessary arthrocentesis or during surgery for joint replacement. PB neutrophils were isolated by dextran sedimentation and hypotonic salt solution as previously described $(8,17)$.

$D M M B$ assay for quantification of sulfated glycoproteins. After differentiation in 3D culture, chondrocytes were treated with NETs for 48 hours, and supernatants were collected to quantify the amount of glycoprotein released as previously described (34). Briefly, 20- $\mu \mathrm{L}$ aliquots of supernatant were added to a microtiter plate, $180 \mu \mathrm{L}$ of DMMB reagent was added, and absorbance was measured at $530 \mathrm{~nm}$. Values were calculated based on standard concentrations of chondroitin sulfate.

Chondrocyte culture. Articular knee chondrocytes (Lonza, CC-2550) were cultured in CGM BulletKit Medium (Lonza, CC-3216) and differentiated using CDM BulletKit Medium (Lonza, CC-3225), TGF- $\beta 3$ (Lonza, PT-4124), ascorbic acid (Lonza, CC-4398), hydrochloric acid, and bovine albumin fraction, following the manufacturer's instructions. Cells were cultured at $37^{\circ} \mathrm{C}$ and $5 \% \mathrm{CO}_{2}$ in a humidified incubator. For $3 \mathrm{D}$ cultures of chondrocytes, approximately 100,000 chondrocytes were resuspended in hydration fluid and embedded in 3D culture using the Cell-Mate3D uGel 40 kit (BRTI Life Sciences). Cells were equilibrated in Chondrocyte Growth Medium (Cell Applications, Inc) and subsequently redifferentiated for 21 days using CDM Chondrocyte Differentiation Media Bullet Kit (Lonza). Media were replenished every 3-4 days.

Aggrecanase-1 activity. Aggrecanase-1 activity was measured in neutrophil lysate, NETs, and supernatants of OA and RA FLSs treated with NETs using the SensoLyte 520 Aggrecanase-1 Fluorimetric Assay Kit (Ana Spec). The assay was performed following Protocol B of the manufacturer's instructions, and the activity is expressed in terms of relative fluorescence units (RFU).

$R N A$-sequencing. RNA from either untreated or NET-treated FLSs was isolated using Direct-zol RNA Miniprep Kit (Zymo Research), and cDNA libraries were prepared with poly(A) tail enrichment using NEBNext Ultra II RNA Library Prep Kit following the manufacturer's instructions (New England Biolabs). RNA-sequencing libraries were sequenced on an Illumina Hiseq 3000 as single end with 50 base pair reads. Reads were demultiplexed and converted to FASTQ using Casava 1.8.2 and then mapped to hg19 using TopHat 2.1.0. Reads per kilobase million mapped reads (RPKM) was calculated using Partek GS 6. ANOVA comparisons were computed on $\log _{2}$-transformed RPKM with a 0.1 offset also using Partek GS. RNA-sequencing data were deposited in the National Center for Biotechnology Information's Gene Expression Omnibus database (accession number GSE150466).

Quantitative PCR. Cells were resuspended in $500 \mu \mathrm{L}$ of TRI Reagent (MilliporeSigma). After $30 \mathrm{~min}$ utes, RNA was isolated using Direct-zol RNA Miniprep Kit (Zymo Research, 11-331) according to the manufacturer's instructions. Total RNA (300 ng) was reverse-transcribed using iScript RT single-strand cDNA (Bio-Rad). Quantitative PCR was performed using TaqMan Gene Expression Master Mix (Thermo Fisher Scientific), human GAPDH primers (Hs99999905_m1), and sequence-specific primers for IL6 (Hs00174131_m1), IL8 (Hs00174103_m1), IL33 (Hs04931857_m1), IL11 (Hs01055414_m1), MMP3 (Hs00968305_m1), PADI2 (Hs01042505_m1), and PADI4 (Hs01057483_m1).

ELISA to quantify NET complexes in SF. The presence of NET complexes in RA and OA SF was assessed by ELISA. A 96-well plate was coated with rabbit polyclonal anti-aggrecanase -1 (Thermo Fisher Scientific, PA1-1750) or anti-aggrecanase-2 (Thermo Fisher Scientific, PA1-1751A) or anti-neutrophil elastase (Calbiochem, 481001) at 1:400 in PBS overnight at $4^{\circ} \mathrm{C}$. Wells were washed 3 times in washing buffer ( $0.05 \%$ Tween in PBS) and blocked in blocking buffer (1\% BSA in PBS) at room temperature (RT) for 1 hour. SFs were diluted 1:100 in blocking buffer and incubated overnight at $4^{\circ} \mathrm{C}$. The wells were washed 3 
times and incubated with mouse monoclonal anti-double-stranded DNA antibody (MilliporeSigma, clone BV16-13, MAB030) at 1:100 in blocking buffer. After washing 3 times, goat anti-mouse conjugated HRP antibody $(1: 10,000)$ (Bio-Rad, 1721012) was incubated for 1 hour at RT. Wells were washed 5 times followed by the addition of TMB substrate (MilliporeSigma) and stop solution (MilliporeSigma). The absorbance was measured at $450 \mathrm{~nm}$, and values were calculated as an OD index.

Autoantibody assessment by ELISA. A 96-well plate was coated with $100 \mathrm{ng} / \mathrm{mL}$ recombinant or citrullinated human recombinant aggrecan, biglycan, or clusterin overnight in PBS at $4^{\circ} \mathrm{C}$. The plate was washed and blocked with $1 \%$ BSA for 1 hour at RT. Serum samples or SF samples from RA subjects or mice were diluted (1:100) and incubated overnight at $4^{\circ} \mathrm{C}$. The plate was washed 3 times in PBS-Tween and incubated with HRP-conjugated anti-human IgG secondary antibody (MilliporeSigma, AQ112P) for 1 hour at RT. After washing 5 times with PBS-Tween, TMB substrate (MilliporeSigma) and stop solution (MilliporeSig$\mathrm{ma}$ ) were used to develop the plate. The absorbance was measured at $450 \mathrm{~nm}$, and values were calculated as an OD index calculated as a ratio of patient to healthy control.

PAD expression by flow cytometry. To quantify the expression of PADs on the membranes of RA and OA FLSs, cells were first blocked with Human TruStain FcX (BioLegend, 422301) at 1:50 dilution in FACS buffer (2\% FBS in PBS) for 10 minutes, then washed in FACS buffer and incubated with either rabbit polyclonal anti-PAD2 (Proteintech, 12110-1-AP) or mouse monoclonal anti-PAD4 (LifeSpan Biosciences, LS-C174028-100) at a 1:50 dilution in FACS buffer for 30 minutes at $4^{\circ} \mathrm{C}$, followed by a wash. The cells were then stained with PE anti-mouse IgG1 (BioLegend, 406607) or PE donkey anti-rabbit IgG (BioLegend, 406421) at 1:100 dilution in FACS buffer for 30 minutes at $4^{\circ} \mathrm{C}$. After washing, cells were fixed in $2 \%$ paraformaldehyde. To quantify the intracellular expression of PADs in RA and OA FLSs, cells were first fixed and permeabilized using the eBioscience Foxp3 kit (Thermo Fisher Scientific) according to the manufacturer's instructions. Staining then proceeded as described above. Isotypes were included for each sample.

In vitro protein degradation assays. RA NETs were preincubated with the indicated concentrations of aggrecanase-1, MMP8, or neutrophil elastase inhibitor for 30 minutes at $37^{\circ} \mathrm{C}$. Subsequently, $1 \mu \mathrm{g}$ recombinant human aggrecan, biglycan, or clusterin was added to the reaction mixture and incubated for $15 \mathrm{~min}$ utes at $37^{\circ} \mathrm{C}$. Following incubation, loading dye was added to samples, and the mixture was incubated for 5 minutes at $95^{\circ} \mathrm{C}$. Samples were then run on a NuPAGE Bis-Tris gel (Invitrogen, Thermo Fisher Scientific) and transferred to a nitrocellulose membrane for Western blot. Blots were washed briefly with water and blocked in $10 \%$ BSA for 30 minutes at RT. Blots were subsequently incubated with the relevant primary antibody (1:1000 dilution) overnight at $4^{\circ} \mathrm{C}$. After incubation in primary antibody, blots were washed 3 times with PBS-Tween and incubated in secondary antibody (diluted in 5\% BSA) for 1 hour. Blots were washed again 3 times and captured using a LI-COR Odyssey CLx scanner.

Generation of mouse FLSs and in vivo administration of FLSS with and without NETs. Breeding pairs of DRB1*04:01 were a gift from C. David (Mayo Clinic, Rochester, Minnesota, USA), and they were housed and bred at the NIH facility. Animal studies were conducted according to the guidelines established by the National Institute of Arthritis and Musculoskeletal and Skin Diseases Laboratory Animal Care and Use section and following an approved protocol (A016-05-26). Eight-week-old DRB1*04:01 female mice were euthanized, and synovium from the tibiofemoral compartment was isolated and dissected. A piece of synovial tissue was incubated in a 12-well plate with CMRL 1066 medium supplemented with 10\% FBS, 1\% L-glutamine, and $1 \%$ penicillin-streptomycin. DRB1*04:01 FLSs were used at passage 4 to ensure purity of the cells. FLSs were cultured in the presence or absence of $50 \mu \mathrm{g}$ of spontaneously formed human RA NETs for 3 days. FLSs were washed with PBS, detached with trypsin, centrifuged at $300 \mathrm{~g}$ for 5 minutes at RT, washed, and resuspended in $1 \times$ Hanks' balanced salt solution. Twelve-week-old DRB1*04:01 female mice were anesthetized using isoflurane vaporization, and the left hind leg was shaved to expose the knee joint. A total of $1 \times 10^{5} \mathrm{DRB} 1^{*} 04: 01$ FLSs with or without uploaded NETs were injected into the synovial space using a 27-gauge needle. This procedure was performed every 14 days for a total of 14 weeks (7 injections), and mice were sacrificed at 24 weeks of age.

Assessment of macrophage activation. To obtain M1 polarized macrophages, human CD14 $4^{+}$monocytes were isolated from PBMCs using positive selection with MACS columns (Miltenyi Biotec), and the cells were incubated with M-CSF (50 ng/mL, PeproTech) for 5 days. Then, 96-well plates were coated with recombinant or citrullinated recombinant human aggrecan or biglycan generated in vitro $(100 \mathrm{ng} / \mathrm{mL})$ in PBS at $4^{\circ} \mathrm{C}$ overnight. Plates were then incubated with $100 \mu \mathrm{g}$ of isolated IgG for 2 hours at RT. Plates were washed twice in PBS and seeded with M1 macrophages for 24 or 72 hours. Collected supernatant was analyzed by commercial ELISAs for TNF- $\alpha$, IL-1 $\beta$, IL-6, and IL-8 (Invitrogen, Thermo Fisher Scientific). 
Detection of citrullinated proteins using $R h-P G$ probe. To perform in vitro citrullination, RA FLS supernatants were incubated in the presence or absence of $1 \mu \mathrm{g}$ recombinant aggrecan, biglycan, clusterin, PAD2 or PAD4 in PBS containing $2 \mathrm{mM} \mathrm{CaCl}_{2}$, and $1 \mathrm{mM} \mathrm{DTT}$. The reaction was incubated at $37^{\circ} \mathrm{C}$ overnight. In vitro citrullinated samples were analyzed using the Rh-PG citrulline probe (Cayman Chemical). Each sample was treated with $20 \%$ trichloroacetic acid and $0.1 \mathrm{mM} \mathrm{Rh}-\mathrm{PG}$ for 30 minutes at $37^{\circ} \mathrm{C}$. Samples were cooled on ice for 30 minutes and centrifuged at $20,817 \mathrm{~g}$ for 5 minutes at $4^{\circ} \mathrm{C}$. The supernatant was removed, and samples were washed with cold acetone and dried at $95^{\circ} \mathrm{C}$ for 1 minute. After resuspending in $50 \mathrm{mM}$ HEPES, samples were separated by SDS-PAGE.

$D R B 1 * 04: 01$ explants in the presence of NET assay. Twelve-week-old DRB ${ }^{*} 04: 01$ were euthanized, and the tibiofemoral compartment was isolated and placed in a 12-well plate with CMRL 1066 medium supplemented with 10\% FBS, 1\% L-glutamine, and 1\% penicillin-streptomycin. Explants were incubated in the presence or absence of spontaneously generated NETs from patients with RA, NETs in the presence of elastase inhibitor, or with $1 \mu \mathrm{g}$ of recombinant human neutrophil elastase at $37^{\circ} \mathrm{C}$. After 2 weeks, explants were fixed in formalin and embedded in parafilm.

Neutrophil elastase activity assay. One hundred microliters of supernatants from control neutrophils treated with either TNF or IL-1 $\beta$ were plated in a 96-well plate. Ten microliters of neutrophil elastase substrate (Z-Ala-Ala-Ala-Ala)2Rh110 (Cayman Chemical) was added to each well, and the plate was incubated for 1.5 hours at $37^{\circ} \mathrm{C}$. Fluorescence was measured in a BioTek Synergy H1 Hybrid Multi-Mode Reader (Agilent). Results are reported in RFU.

Cloning of aggrecan-specific T cells. Cit-aggrecan-specific RA T cell clones restricted by HLA-DRB*0401 were obtained as previously described (6).

FLS culture. Human OA and RA FLSs were isolated as previously described (35). Cells were grown in CMRL medium (Invitrogen, Thermo Fisher Scientific) supplemented with 10\% FBS (Invitrogen, Thermo Fisher Scientific), $2 \mathrm{mM}$ glutamine, penicillin $(100 \mathrm{U} / \mathrm{mL})$, and streptomycin $(100 \mu \mathrm{g} / \mathrm{mL})$. Cells were cultured at $37^{\circ} \mathrm{C}$ and $5 \% \mathrm{CO}_{2}$ in a humidified incubator.

NET isolation. NETs were isolated, as previously described (17). Briefly, RA neutrophils were purified and seeded in 24-well tissue culture plates in RPMI without phenol or stimuli and incubated for 4 hours at $37^{\circ} \mathrm{C}$. Supernatants were harvested with micrococcal nuclease $(10 \mathrm{U} / \mathrm{mL}$; Thermo Fisher Scientific) for 15 minutes at $37^{\circ} \mathrm{C}$. Supernatants were collected and centrifuged at $300 \mathrm{~g}$ for 5 minutes at $4^{\circ} \mathrm{C}$. NET supernatants were transferred to a fresh tube and stored at $-80^{\circ} \mathrm{C}$ until used.

IgG purification. IgGs were purified from RA or OA SF samples with a Melon Gel IgG Spin kit following the manufacturer's instructions (Thermo Fisher Scientific).

Assessment of FLS antigen presentation to antigen-specific T cell clones. The ability of FLSs to present citrullinated antigens to $\mathrm{T}$ cells was assessed as previously described (17). Briefly, FLSs were pretreated with recombinant IFN- $\gamma$ to upregulate MHC II. Three days later, cells were washed twice with PBS and then plated at a density of 100,000 cells per well in human T cell medium. CellTrace violet-labeled (Thermo Fisher Scientific) human cit-aggrecan-specific T cells were added to FLS cultures at a ratio of 1:2 (FLS/T cell) and incubated in the presence or absence of their specific citrullinated peptide $(2 \mu \mathrm{g})$. Supernatants were then collected and centrifuged at $300 \mathrm{~g}$ for 5 minutes at RT. IFN- $\gamma$ was quantified using ELISA ReadySET-Go! (eBioscience, Thermo Fisher Scientific) according to the manufacturer's recommendations. CellTrace-labeled $\mathrm{T}$ cells were used to assess proliferation by flow cytometry.

Western blot. Cells were resuspended in lysis buffer (36) and placed at $4^{\circ} \mathrm{C}$ on a rotator for approximately 30 minutes. Lysate was centrifuged for 10 minutes at $20,817 \mathrm{~g}$ at $4^{\circ} \mathrm{C}$. The supernatants were transferred to a fresh Eppendorf tube. Total protein was quantified using BCA assay (Thermo Fisher Scientific). Equal amounts of total protein were resolved in a 4\%-12\% gradient Bis-Tris gel (Invitrogen, Thermo Fisher Scientific), transferred onto a nitrocellulose membrane, and blocked with 10\% BSA for 30 minutes at RT. After overnight incubation with primary antibodies, membranes were washed 3 times with PBS-Tween and incubated with secondary antibody coupled to IRDye 800CW. Membranes were developed using LI-COR Odyssey CLx scanner.

Statistics. Data were analyzed using GraphPad Prism software. For samples with non-Gaussian distribution, we used Student's 2-tailed Mann-Whitney $U$ test. Multiple comparisons were analyzed by Kruskal-Wallis 1-way ANOVA test with post hoc Dunn's test. Results are presented as the means \pm SEM. $P$ values of less than 0.05 were considered significant.

Study approval. All individuals gave written informed consent and were enrolled in a protocol approved by the National Institute of Arthritis and Musculoskeletal and Skin Diseases/National Institute of Diabe- 
tes and Digestive and Kidney Diseases IRB (protocol 01-AR-0227) or the University of Michigan Medical IRB (protocol HUM00043667 or HUM00045058). The study was conducted according to the standards of the Declaration of Helsinki.

Animal studies were conducted according to the guidelines established by the National Institute of Arthritis and Musculoskeletal and Skin Diseases (NIAMS) Laboratory Animal Care and Use section and following an approved protocol (A016-05-26).

\section{Author contributions}

CCR, PMC, and RRG performed the experiments; CCR, PMC, RRG, and SRB analyzed the data and performed statistical analyses; VH analyzed and scored tissue slides; and EJ, CR, DAF, and JHB provided specimens and clinical information. CCR, PMC, and MJK were involved in overall design and manuscript preparation. CCR, PMC, and MJK drafted the manuscript.

\section{Acknowledgments}

This study was supported by the Intramural Research Program, NIAMS/NIH, ZIA AR041199.

Address correspondence to: Carmelo Carmona-Rivera or Mariana J. Kaplan, Systemic Autoimmunity Branch, NIAMS, NIH, 10 Center Drive, 12S 253 (CCR) or 12N248C (MJK), Bethesda, Maryland 20892, USA. Phone: 301.827.9527; Email: carmelo.carmona-rivera@nih.gov (CCR). Phone: 301.496.0517; Email: mariana.kaplan@nih.gov (MJK).

1. Holers VM. Autoimmunity to citrullinated proteins and the initiation of rheumatoid arthritis. Curr Opin Immunol. 2013;25(6):728-735

2. Aggarwal A, Srivastava R, Agrawal S. T cell responses to citrullinated self-peptides in patients with rheumatoid arthritis. Rheumatol Int. 2013;33(9):2359-2363.

3. Glant TT, et al. Characterization and localization of citrullinated proteoglycan aggrecan in human articular cartilage. PLoS One. 2016;11(3):e0150784.

4. Law SC, et al. T-cell autoreactivity to citrullinated autoantigenic peptides in rheumatoid arthritis patients carrying HLA-DRB1 shared epitope alleles. Arthritis Res Ther. 2012;14(3):R118.

5. Markovics A, Ocskó T, Katz RS, Buzás EI, Glant TT, Mikecz K. Immune recognition of citrullinated proteoglycan aggrecan epitopes in mice with proteoglycan-induced arthritis and in patients with rheumatoid arthritis. PLoS One. 2016;11(7):e0160284.

6. Rims C, et al. Citrullinated aggrecan epitopes as targets of autoreactive CD4+ T cells in patients with rheumatoid arthritis. Arthritis Rheumatol. 2019;71(4):518-528.

7. Pillinger MH, Abramson SB. The neutrophil in rheumatoid arthritis. Rheum Dis Clin North Am. 1995;21(3):691-714.

8. Khandpur R, et al. NETs are a source of citrullinated autoantigens and stimulate inflammatory responses in rheumatoid arthritis. Sci Transl Med. 2013;5(178):178ra40.

9. Sur Chowdhury C, Giaglis S, Walker UA, Buser A, Hahn S, Hasler P. Enhanced neutrophil extracellular trap generation in rheumatoid arthritis: analysis of underlying signal transduction pathways and potential diagnostic utility. Arthritis Res Ther. 2014;16(3):R122.

10. Arner EC. Aggrecanase-mediated cartilage degradation. Curr Opin Pharmacol. 2002;2(3):322-329.

11. Verma P, Dalal K. ADAMTS-4 and ADAMTS-5: key enzymes in osteoarthritis. J Cell Biochem. 2011;112(12):3507-3514.

12. Yamanishi Y, et al. Expression and regulation of aggrecanase in arthritis: the role of TGF-beta. J Immunol. 2002;168(3):1405-1412.

13. Papayannopoulos V, Metzler KD, Hakkim A, Zychlinsky A. Neutrophil elastase and myeloperoxidase regulate the formation of neutrophil extracellular traps. J Cell Biol. 2010;191(3):677-691.

14. Bawadekar M, et al. Peptidylarginine deiminase 2 is required for tumor necrosis factor alpha-induced citrullination and arthritis, but not neutrophil extracellular trap formation. J Autoimmun. 2017;80:39-47.

15. Damgaard D, Senolt L, Nielsen CH. Increased levels of peptidylarginine deiminase 2 in synovial fluid from anti-CCP-positive rheumatoid arthritis patients: Association with disease activity and inflammatory markers. Rheumatology (Oxford). 2016;55(5):918-927

16. Kinloch A, et al. Synovial fluid is a site of citrullination of autoantigens in inflammatory arthritis. Arthritis Rheum. 2008;58(8):2287-2295.

17. Carmona-Rivera C, et al. Synovial fibroblast-neutrophil interactions promote pathogenic adaptive immunity in rheumatoid arthritis. Sci Immunol. 2017;2(10):eaag3358.

18. Bläss S, Engel JM, Burmester GR. The immunologic homunculus in rheumatoid arthritis. Arthritis Rheum. 1999;42(12):2499-2506

19. Gregersen PK, Silver J, Winchester RJ. The shared epitope hypothesis. An approach to understanding the molecular genetics of susceptibility to rheumatoid arthritis. Arthritis Rheum. 1987;30(11):1205-1213.

20. Chang MH, Nigrovic PA. Antibody-dependent and -independent mechanisms of inflammatory arthritis. JCI Insight. 2019;4(5):125278.

21. Mathsson L, Lampa J, Mullazehi M, Rönnelid J. Immune complexes from rheumatoid arthritis synovial fluid induce FcgammaRIIa dependent and rheumatoid factor correlated production of tumour necrosis factor-alpha by peripheral blood mononuclear cells. Arthritis Res Ther. 2006;8(3):R64. 
22. Monach PA, et al. A broad screen for targets of immune complexes decorating arthritic joints highlights deposition of nucleosomes in rheumatoid arthritis. Proc Natl Acad Sci US A. 2009;106(37):15867-15872.

23. Chen W, Wang Q, Ke Y, Lin J. Neutrophil function in an inflammatory milieu of rheumatoid arthritis. J Immunol Res. 2018;2018:8549329.

24. Nagase H, Kashiwagi M. Aggrecanases and cartilage matrix degradation. Arthritis Res Ther. 2003;5(2):94-103.

25. Vankemmelbeke MN, et al. Expression and activity of ADAMTS-5 in synovium. Eur J Biochem. 2001;268(5):1259-1268.

26. Yamanishi Y, et al. Expression and regulation of aggrecanase in arthritis: the role of TGF-beta. J Immunol. 2002;168(3):1405-1412.

27. Keiser H, Greenwald RA, Feinstein G, Janoff A. Degradation of cartilage proteoglycan by human leukocyte granule neutral proteases--a model of joint injury. II. Degradation of isolated bovine nasal cartilage proteoglycan. J Clin Invest. 1976;57(3):625-632.

28. Hilbert N, Schiller J, Arnhold J, Arnold K. Cartilage degradation by stimulated human neutrophils: elastase is mainly responsible for cartilage damage. Bioorg Chem. 2002;30(2):119-132.

29. van Vulpen LF, et al. IL-1 $\beta$, in contrast to TNF $\alpha$, is pivotal in blood-induced cartilage damage and is a potential target for therapy. Blood. 2015;126(19):2239-2246.

30. Zwerina J, et al. TNF-induced structural joint damage is mediated by IL-1. Proc Natl Acad Sci U S A. 2007;104(28):11742-11747.

31. Byrd AS, et al. Neutrophil extracellular traps, B cells, and type I interferons contribute to immune dysregulation in hidradenitis suppurativa. Sci Transl Med. 2019;11(508):eaav5908

32. Salinger AJ, et al. Technical comment on "Synovial fibroblast-neutrophil interactions promote pathogenic adaptive immunity in rheumatoid arthritis”. Sci Immunol. 2020;5(43):eaax5672.

33. Villeneuve E, Nam J, Emery P. 2010 ACR-EULAR classification criteria for rheumatoid arthritis. Rev Bras Reumatol. 2010;50(5):481-483.

34. Mort JS, Roughley PJ. Measurement of glycosaminoglycan release from cartilage explants. Methods Mol Med. 2007;135:201-209.

35. Tran CN, et al. Presentation of arthritogenic peptide to antigen-specific T cells by fibroblast-like synoviocytes. Arthritis Rheum. 2007;56(5):1497-1506

36. Carmona-Rivera C, Simeonov DR, Cardillo ND, Gahl WA, Cadilla CL. A divalent interaction between HPS1 and HPS4 is required for the formation of the biogenesis of lysosome-related organelle complex-3 (BLOC-3). Biochim Biophys Acta. 2013;1833(3):468-478. 